\title{
Severe Candida glabrata pancolitis and fatal Aspergillus fumigatus pulmonary infection in the setting of bone marrow aplasia after CD19-directed CAR T-cell therapy - a case report
}

Kai Rejeski ${ }^{1,2,3^{*}}$ (D), Wolfgang G. Kunz ${ }^{4}$, Martina Rudelius ${ }^{5}$, Veit Bücklein ${ }^{1,2}$, Viktoria Blumenberg ${ }^{1,2}$, Christian Schmidt ${ }^{1}$, Philipp Karschnia ${ }^{6}$, Florian Schöberl ${ }^{7}, K^{\prime}$ Konstantin Dimitriadis ${ }^{7}$, Louisa von Baumgarten ${ }^{7}$, Joachim Stemmler ${ }^{1}$, Oliver Weigert ${ }^{1}$, Martin Dreyling ${ }^{1}$, Michael von Bergwelt-Baildon ${ }^{1,3}$ and Marion Subklewe $e^{1,2,3}$

\begin{abstract}
Background: Prolonged myelosuppression following CD19-directed CAR T-cell transfusion represents an important, yet underreported, adverse event. The resulting neutropenia and multifactorial immunosuppression can facilitate severe infectious complications.

Case presentation: We describe the clinical course of a 59-year-old patient with relapsed/refractory DLBCL who received Axicabtagene-Ciloleucel (Axi-cel). The patient developed ASTCT grade I CRS and grade IV ICANS, necessitating admission to the neurological ICU and prolonged application of high-dose corticosteroids and other immunosuppressive agents. Importantly, neutropenia was profound (ANC < 100/ $\mathrm{Il)}$, G-CSF-refractory, and prolonged, lasting more than 50 days. The patient developed severe septic shock 3 weeks after CAR transfusion while receiving anti-fungal prophylaxis with micafungin. His clinical status stabilized with broad anti-infective treatment and intensive supportive measures. An autologous stem cell backup was employed on day 46 to support hematopoietic recovery. Although the counts of the patient eventually started to recover, he developed an invasive pulmonary aspergillosis, which ultimately lead to respiratory failure and death. Postmortem examination revealed signs of Candida glabrata pancolitis.

(Continued on next page)
\end{abstract}

\footnotetext{
*Correspondence: kai.rejeski@med.uni-muenchen.de

'Department of Hematology and Oncology, University Hospital, LMU

Munich, Munich, Germany

${ }^{2}$ Laboratory for Translational Cancer Immunology, LMU Gene Center, Munich, Germany

Full list of author information is available at the end of the article
}

(C) The Author(s). 2021 Open Access This article is licensed under a Creative Commons Attribution 4.0 International License, which permits use, sharing, adaptation, distribution and reproduction in any medium or format, as long as you give appropriate credit to the original author(s) and the source, provide a link to the Creative Commons licence, and indicate if changes were made. The images or other third party material in this article are included in the article's Creative Commons licence, unless indicated otherwise in a credit line to the material. If material is not included in the article's Creative Commons licence and your intended use is not permitted by statutory regulation or exceeds the permitted use, you will need to obtain permission directly from the copyright holder. To view a copy of this licence, visit http://creativecommons.org/licenses/by/4.0/ The Creative Commons Public Domain Dedication waiver (http://creativecommons.org/publicdomain/zero/1.0/) applies to the data made available in this article, unless otherwise stated in a credit line to the data. 


\begin{abstract}
(Continued from previous page)
Conclusions: This case highlights the increased risk for fatal infectious complications in patients who present with profound and prolonged cytopenia after CAR T-cell therapy. We describe a rare case of C. glabrata pancolitis associated with multifactorial immunosuppression. Although our patient succumbed to a fatal fungal infection, autologous stem cell boost was able to spur hematopoiesis and may represent an important therapeutic strategy for DLBCL patients with CAR T-cell associated bone marrow aplasia who have underwent prior stem cell harvest.
\end{abstract}

Keywords: CAR T-cell, Case report, Hematotoxicity, Candida glabrata, Invasive aspergillosis

\section{Background}

When CD19-directed Chimeric Antigen Receptor (CAR) $\mathrm{T}$-cell therapies were first introduced into the clinical setting, a set of distinctive side effects were observed, including Cytokine Release Syndrome (CRS) and immune effector cell-associated neurotoxicity syndrome (ICANS). As these treatments transition from large clinical trials to real-world implementation, new nuances of the side effect spectrum have emerged. This includes the observation that patients can present not only with grade $3-4$ cytopenia (CTCAE v5.0), but also a syndrome of persistent cytopenia after CAR $T$-cell transfusion $[1,2]$. Hematologic toxicity is common with one study demonstrating that neutropenia, thrombocytopenia and anemia occur in 94,80 and $51 \%$ of patients respectively [3]. Risk factors for hematotoxicity include CRS grade, baseline cytopenia, and prior allogeneic stem cell transplantation within the last year $[2,3]$. The fact that baseline hematopoietic reserve contributes to cytopenia in CAR $\mathrm{T}$-cell patients may explain the variability in the incidence of hematoxicity in the real-world setting compared to the large registration trials, which set stringent hematologic exclusion criteria $[4,5]$. The clinical utility of therapeutic rescue strategies that mitigate the risk of infectious complications secondary to long-term bone marrow (BM) aplasia, such as early autologous stem cell boost or Eltrombopag, remains unknown. Moreover, the relative paucity of reports concerning severe and lifethreatening infections in the reported CD19-directed CAR T-cell trials necessitates the presentation of distinctive clinical courses that underline the risks of persistent cytopenia after CAR T-cell therapy in a realworld setting.

\section{Case presentation}

The 59-year-old male patient was initially diagnosed with stage IIIA follicular lymphoma in January 2018, which transformed to germinal center B-cell like DLBCL in October of the same year. Refractory to multiple cycles of Rituximab-based immunochemotherapy (Fig. 1a), the patient underwent leukapheresis for CAR T-cell therapy in April 2019. The patient experienced significant lymphoma progression during the manufacturing process of Axicabtagene ciloleucel (Axi-cel), manifesting itself in the form of malignant pleural effusions and progressive lymphadenopathy (Fig. 2a,c,e). In the six-week period between leukapheresis and lymphodepletion, the patient remained severely neutropenic after R-CHOP and developed possible invasive fungal disease (IFD) [6] (Fig. 3e) as well as E. coli septicemia secondary to a urinary tract infection. These infections were treated successfully with liposomal amphotericin B and piperacillin/ tazobactam. Prior to lymphodepletion (D-5), the patient presented with an ECOG performance status of 1, intact renal and liver function, while observing residually depressed blood counts (WBC $1270 / \mu \mathrm{l}$, ANC 700/ $\mu$ l, ALC 390/ $\mu$ l, Hemoglobin $7.2 \mathrm{~g} / \mathrm{dl}$, Platelets $20 \mathrm{G} / \mathrm{l}$ ). BM histopathology showed a hypocellular marrow with no evidence of lymphoma infiltration. Analysis of lymphocyte subpopulations demonstrated severe B-cell aplasia and decreased absolute CD4+ $(84 / \mu \mathrm{l})$ and CD8+ $(271 / \mu \mathrm{l})$ counts. His anti-infective prophylaxis consisted of a combination of acyclovir, sulfamethoxazole/trimethoprim (TMP/SMZ), and posaconazole. The fludarabine/ cyclophosphamide lympho-preparative regimen was applied on days -5 to -3 according to the provider's protocol, followed by CAR T-cell transfusion on day 0 .

During lymphodepletion the patient developed an upper respiratory infection, presenting with a nonproductive cough and a sputum culture positive for Bordetella bronchiseptica, which resolved clinically after antibiotic treatment with piperacillin/tazobactam. On day 7 , the patient developed fever with a stable cardiopulmonary function and concomitant IL-6 rise (Fig. 1d), which was classified as CRS grade I according to ASTCT consensus grading [7] in the absence of positive blood cultures and other foci. Due to persistent fever refractory to antipyretic treatment, four total doses of tocilizumab $(8 \mathrm{mg} / \mathrm{kg})$ were applied over $36 \mathrm{~h}$. Starting on day 9 , the patient deteriorated neurologically with a depressed state of consciousness prompting the diagnosis of grade IV ICANS. The patient was transferred to the neurological ICU, and high-dose corticosteroids (methylprednisolone $1000 \mathrm{mg}$ daily) were initiated. CSF-analysis displayed subtle pleocytosis, but revealed no signs of CNS infection (viral, bacterial and fungal) or meningeal involvement of the DLBCL. CT and MR imaging of the brain exhibited none of the pathological findings reported for 


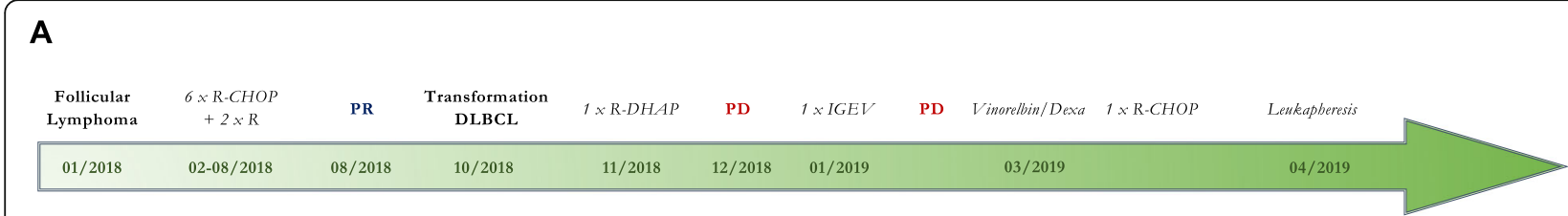

B

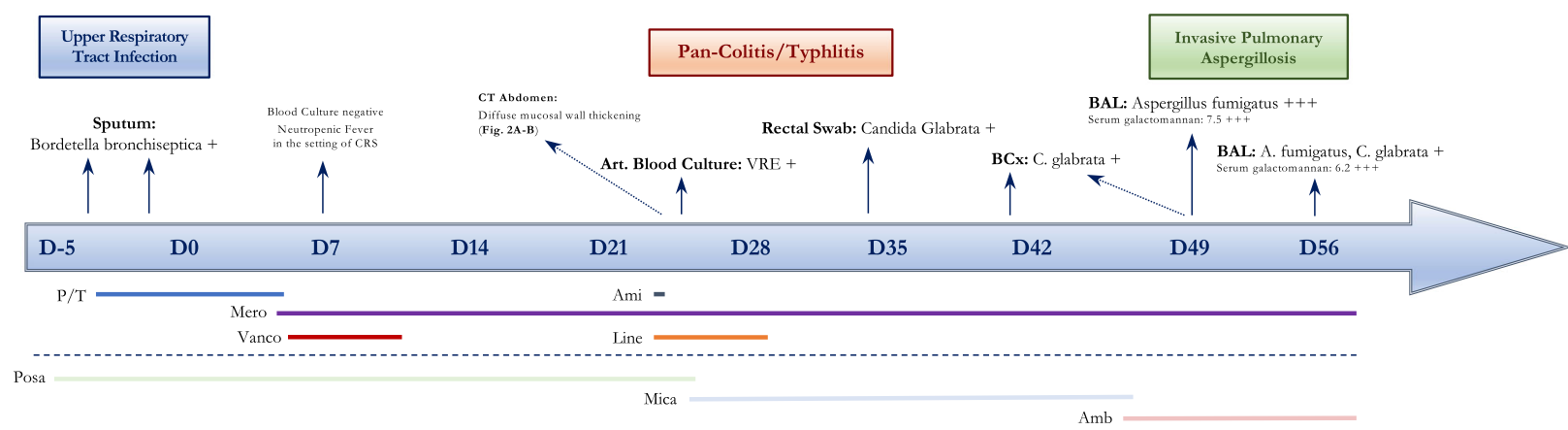

C

\section{D}
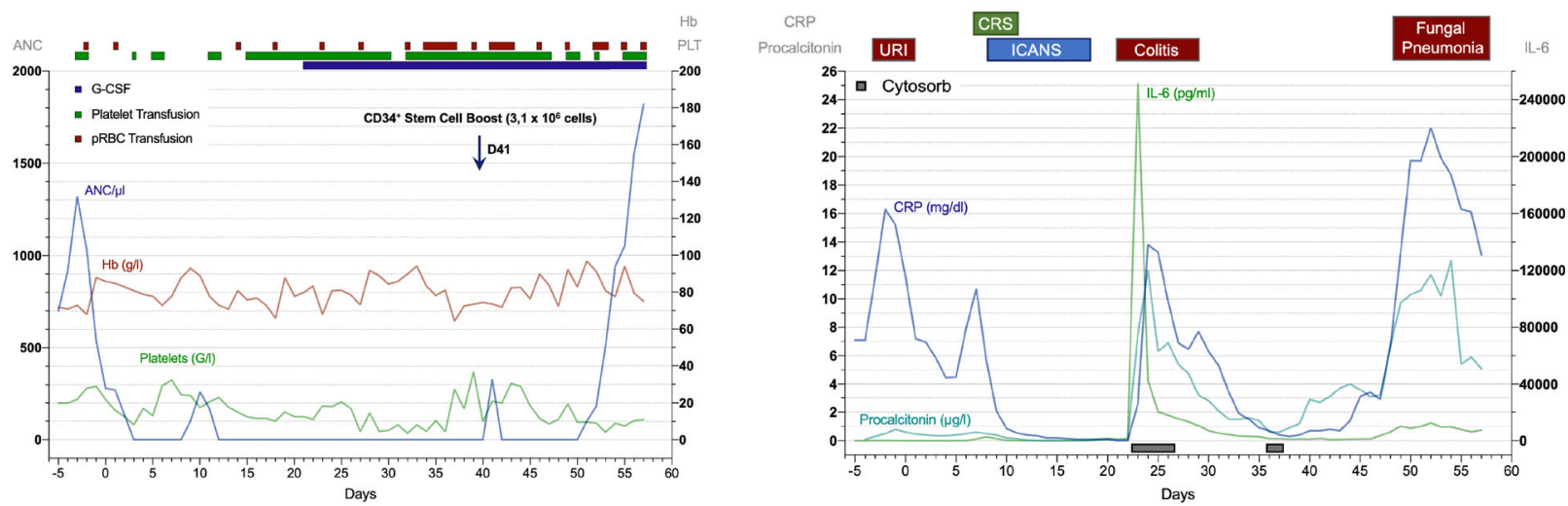

E
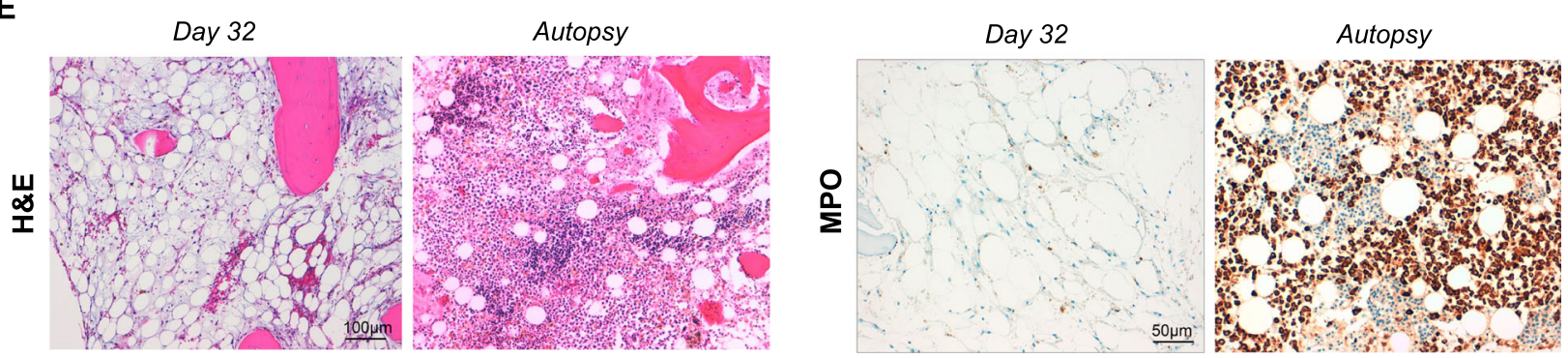

Fig. 1 Overview of CAR T-cell mediated hematotoxicity and infectious complications. a Treatment course prior to CAR T-cell therapy. b Timeline of infectious complications during CAR T-cell treatment course. Positive microbiologic culture results are underlined. Bottom: Overview of utilized anti-infectives with the respective bars displaying the length of treatment. $\mathrm{P} / \mathrm{T}=$ piperacillin/tazobactam, $\mathrm{Ami}=$ amikacin, Mero = meropenem, Vanco $=$ vancomycin, Line $=$ linezolid, Posa $=$ posaconazole (p.o.), Mica $=$ micafungin (i.v.), Amb = liposomal amphotericin B. The patient also received prophylactic acyclovir and sulfamethoxazole/trimethoprim (TMP/SMZ) during treatment. c Complete Blood Count (CBC) timeline. ANC (blue), platelet count (green), Hemoglobin (red). Transfusion events (green: platelet transfusion, red: pRBC transfusion) and G-CSF support (blue bar) are integrated in the curve. $\mathbf{d}$ Dynamics of Serum Inflammatory Markers. Infectious complications are superimposed above the graph. e Histopathologic analysis of BM biopsies demonstrating severe BM aplasia 1 month after CAR T-cell transfusion (Day 32, upper panel) and evidence of recovering hematopoiesis following autologous stem cell transfer (Autopsy, lower panel). Immunohistochemical staining for myeloperoxidase (= MPO) highlighting strong activation of myelopoiesis (right panel) 


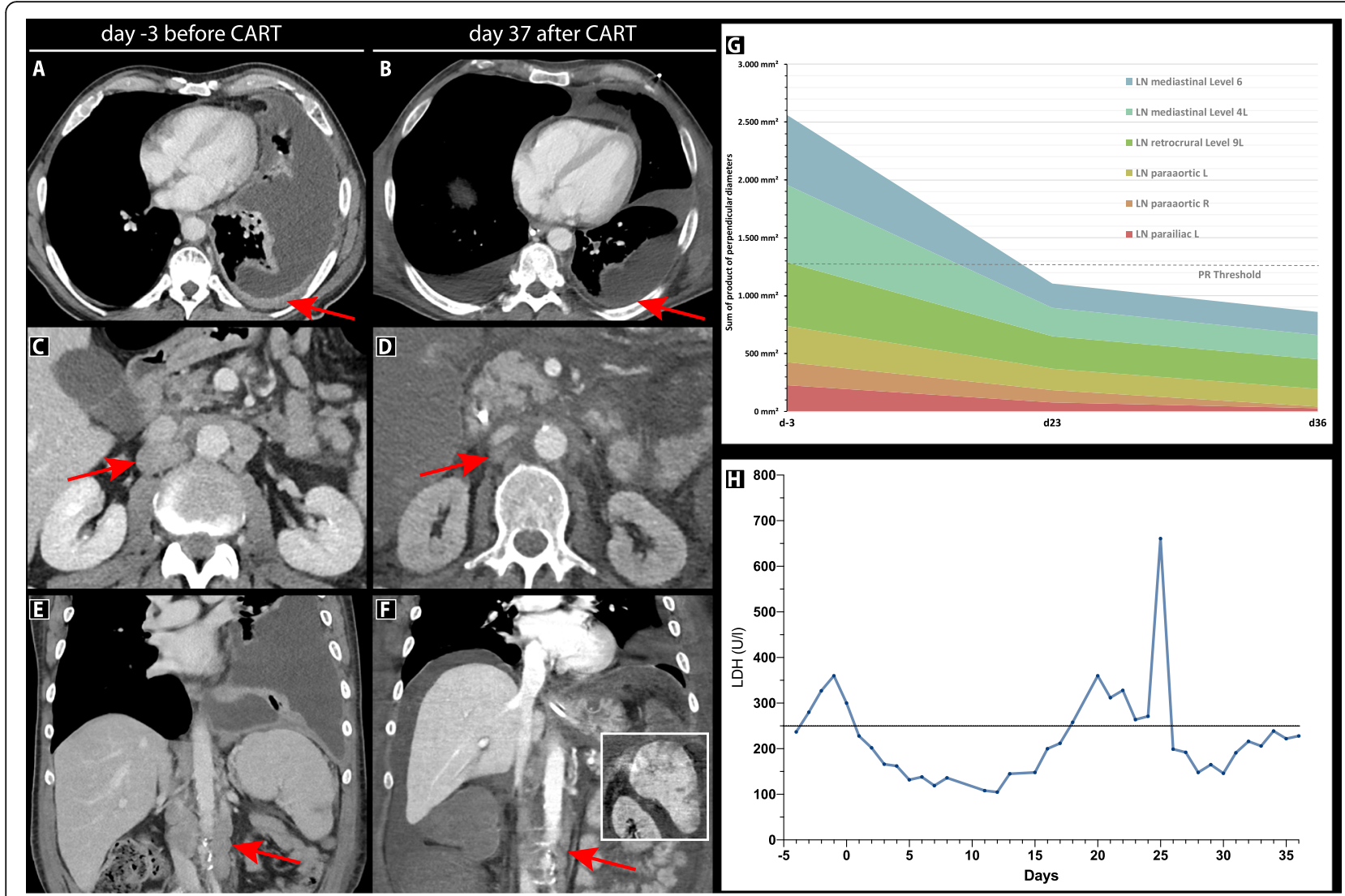

Fig. 2 Radiographic evidence of partial response to Axi-cel after 36 days. Contrast-enhanced CT abdomen (axial view) displaying pleural manifestations before (a) and after (b) CAR T-cell treatment. Involved paraaortic lymph nodes (red arrows) demonstrate an interval decrease in size (c-d, e-f). The inset of panel F depicts splenic hypodensities consistent with necrosis of tumor tissue secondary to immune therapy and a reduction of splenomegaly. G Graphical depiction of response over time. Tumor extent was quantified as the sum of the product of perpendicular diameters according to Lugano criteria over 3 time points and is graphed on the $y$-axis. $\mathbf{H}$ Timeline of LDH levels (U/I). The increase in LDH levels at day 25 correlated with the gastrointestinal toxicity and liver dysfunction

ICANS [8] or CNS infection [9](Fig. 3c-d). After 5 days of high-dose methylprednisolone, the patient's neurological status slowly improved, and corticosteroids could be tapered.

\section{Partial response to Axicabtagene-Ciloleucel after $\mathbf{3 6}$ days according to Lugano criteria}

While the patient's initial clinical course was fraught with complications, CT staging performed at day 36 demonstrated a partial response to CAR T-cell therapy according to Lugano and Lyric criteria (Fig. 2a-g). This was particularly evident for the pleural manifestations of the underlying DLBCL, which was accompanied by an interval decrease in pleural effusions and subjective dyspnea (Fig. 2a-b). Multiple nodal sites including paraaortic (Fig. 2c-d) and parailiac lymph nodes (Fig. 2e-f) exhibited significant regression of tumor size (Fig. 2g). Moreover, the patient displayed splenic hypodensities consistent with either tumor tissue necrosis or infectionassociated inflammatory changes (inset, Fig. 2f). These
CT-morphologic findings were accompanied by a decrease of LDH levels (Fig. 2h).

\section{Prolonged cytopenia lead to the development of severe neutropenic colitis and fatal fungal pneumonia}

The patient presented with significant myelotoxicity and sustained transfusion dependency after CAR T-cell administration (Fig. 1c). The overall duration of profound neutropenia - defined as an ANC $<100 / \mu l[10]$ - was 52 days. Because of the severity of ICANS, the clinicians chose to hold G-CSF during this time period due to concerns for risk of worsening toxicity [11].

While receiving broad anti-infectives (acyclovir, TMP/ SMZ, posaconazole, meropenem), the patient developed breakthrough septic shock on day 24 . He exhibited fever $\left(39.5^{\circ} \mathrm{C}\right)$, hypotension $(70 / 30 \mathrm{mmHg})$, tachycardia $(160 /$ $\mathrm{min}$ ), as well as lactic acidosis (lactate: $10.7 \mathrm{mmol} / \mathrm{l}$ ). The patient was non-conversant, non-oriented, and was not able to localize symptoms. However, he had described epigastric tenderness and subtle changes in stool 


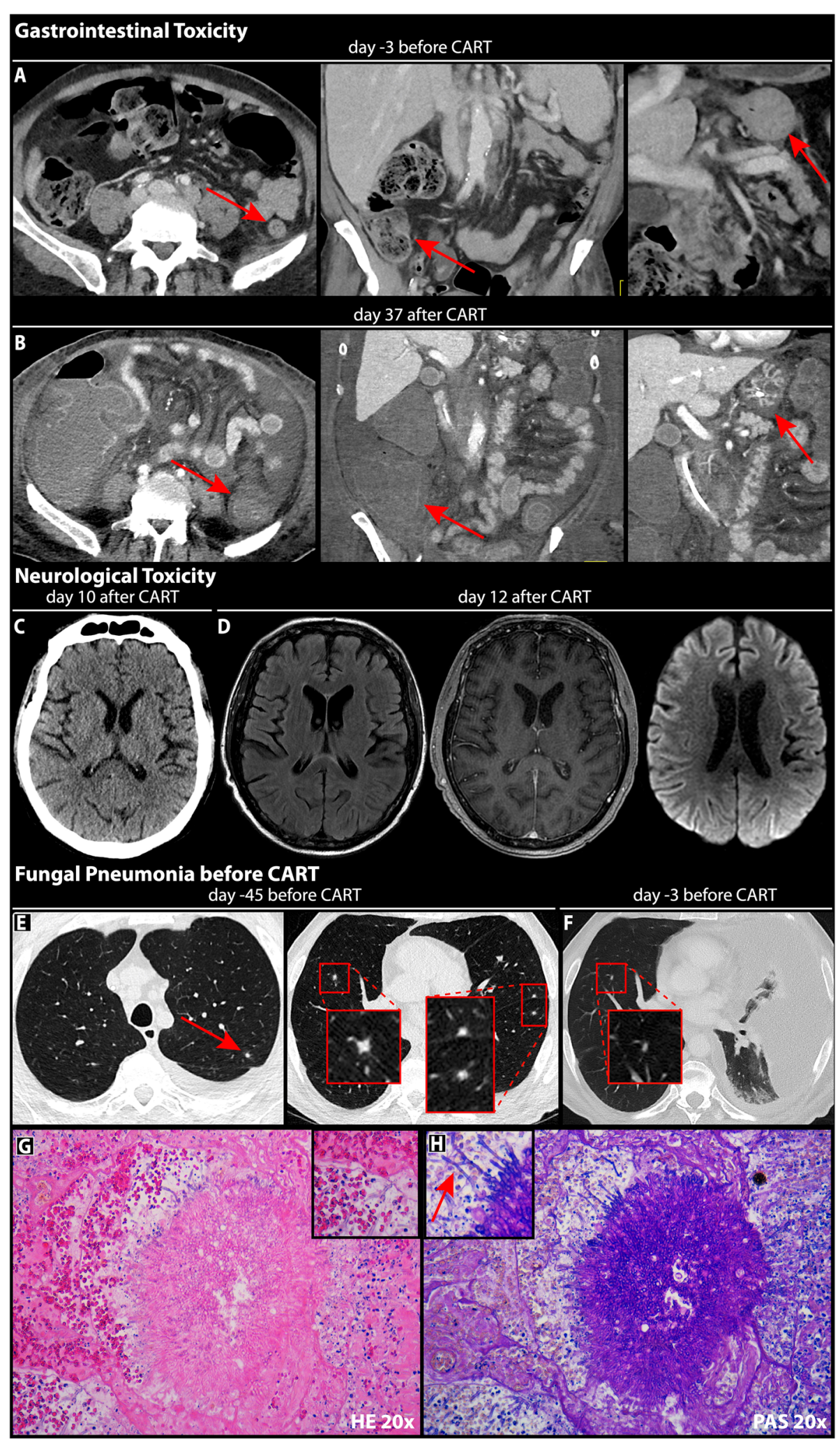

Fig. 3 (See legend on next page.) 


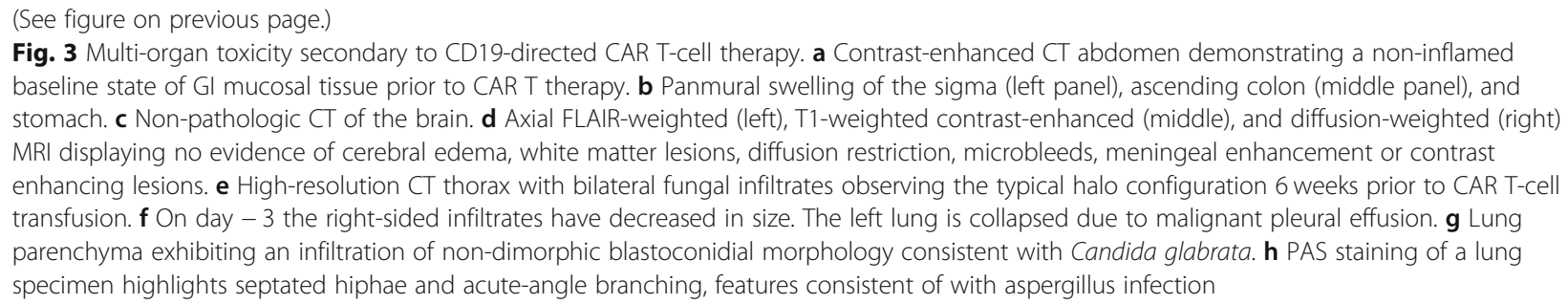

consistency and frequency on the previous day. As a result, abdominal CT imaging was obtained, which displayed severe pancolitis, as evidenced by pronounced mucosal thickening with edematous changes ranging from the ascending colon to the rectum (Fig. 3a-b). Labs were notable for an acute spike of serum inflammatory parameters (Il-6 > 500.000 pg/ml, CRP $5.7 \mathrm{mg} / \mathrm{dl}$, Procalcitonin $11.2 \mathrm{ng} / \mathrm{ml}$, Fig. 1d). Following ICU transfer, high-dose vasopressor support with norepinephrine was initiated together with volume resuscitation to maintain organ perfusion. Due to progressive loss of consciousness the patient was intubated and mechanically ventilated. To control the proinflammatory state, extracorporeal cytokine absorption (Cytosorb ${ }^{\circledR}$ ) was performed, which resulted in a decrease of soluble inflammatory markers in the subsequent days. Arterial blood cultures were positive for vancomycin-resistant enterococci (VRE), and the antibiotic treatment was escalated to include linezolid along with meropenem (Fig. 1b). The ICU team transitioned antifungal prophylaxis from posaconazole to an echinocandine (micafungin $50 \mathrm{mg}$ IV) due to ECG changes and concerns for drug interactions on day 24. Though he remained in critical condition, in the coming weeks his clinical condition stabilized and both the catecholamine rate and volume support could be reduced. To determine the origin of sustained myelosuppression, a bone marrow biopsy was obtained, which depicted a hypocellular marrow and severe aplasia affecting all hematopoietic lineages (Fig. 1e). On days 41 and 49, surveillance blood cultures were positive for Candida glabrata, a commensal fungal organism of human mucosal tissues [12] harboring an intrinsic azole resistance [13]. Susceptibility testing confirmed the azole-resistance of $C$. glabrata isolates. The antimycotic therapy was escalated from micafungin to liposomal amphotericin B. C. glabrata likely disseminated to the bloodstream secondary to the described gastrointestinal toxicity and was previously discovered on rectal swab (Fig. 1b) and fecal culture (day -51).

Due to sustained neutropenia, BM aplasia and the availability of a suitable apheresis product, the CAR Tcell Taskforce proceeded with a stem cell boost of previously collected autologous CD34+ cells $\left(3,1 \times 10^{6}\right.$ cells $)$ on day 46. The combination of a sudden rise in serum inflammatory markers and a strongly positive serum galactomannan (GM) assay on day 49 prompted diagnostic bronchoscopy, which revealed a diffuse infiltrate of alveolar and peri-bronchial tissue by Aspergillus fumigatus, consistent with fulminant invasive fungal pneumonia. Over the next week, the patient became increasingly hemodynamically unstable with poor peripheral oxygenation. Repeat bronchoscopy with bronchial lavage was positive for A. fumigatus and C. glabrata. After 50 days of G-CSF refractory aplasia, the autologous stem cells slowly engrafted leading to neutrophil recovery. However, this likely exacerbated the local inflammatory reaction in the lung, contributing to rapid deterioration of his respiratory status. Despite extensive supportive measures, the patient died on day 58 after CAR T-cell transfusion. Cause of death was confirmed by autopsy as invasive fungal pneumonia with multiorgan septic spread. Post-mortem examination revealed Candida spores in intestinal, colonic and rectal tissue. Of note, histopathologic BM analysis revealed signs of burgeoning hematopoietic regeneration (Fig. 1e).

\section{Discussion and conclusions}

Recent studies have shed light on the high incidence of prolonged and profound neutropenia after adoptive immunotherapy with CD19-specific CAR T-cells [1-3]. Clinical sequalae include severe infectious complications, which have emerged as the number one cause of longterm non-relapse mortality after CAR T-cell therapy [14]. In a seminal study, Hill and colleagues demonstrate that infectious complications are common with a cumulative incidence of $23 \%$ in the first 28 days after CAR Tcell transfusion and $14 \%$ between days 29-90. While bacterial infections were most common in the first month, viral infections - especially respiratory viruses predominated after day 29 , likely as a result of an impaired adaptive immune response due to B-cell aplasia [15]. Overall, most infections were mild to moderate and fatal infections were rare. Invasive mold infections were uncommon, ranging from 1 to $7 \%$ in CAR T-cell recipients [15-17]. In multivariate analyses, the use of systemic corticosteroids for the management of CRS or ICANS has emerged as a major risk factor of infection [18]. Certain inflammatory signatures - such as the 
"double peaks of IL-6" pattern observed in our patient have been shown to confer an especially high risk of lifethreatening infection [19].

This case highlights the combination of factors that precipitate a high risk of infectious complications in a CAR T-cell patient. First, the heavily pre-treated patient displayed B-cell aplasia and a diminished hematopoietic reserve (e.g. trilineage cytopenia) prior to lymphodepletion. Second, the patient developed classic complications of CAR T-cell therapy such as CRS and severe and prolonged ICANS, reflecting a pro-inflammatory state that represents an important risk factor for developing infections in CAR T-cell patients [16]. The subsequent application of high-dose corticosteroids impaired both innate and adaptive immunity [20]. Third, pancytopenia was profound, prolonged and not able to be reverted by growth factor support. Fourth, the patient had a history of infectious complications, including a course of fungal pneumonia 6 weeks prior to lymphodepletion, likely flaring up in the setting of the above three factors.

Our patient developed severe septic shock secondary to neutropenic colitis on day 24, highlighting the risk of a watch-and-wait approach to prolonged neutropenia after CAR T-cell therapy. A two-pronged approach to management appears prescient. On the one hand, measures to propagate hematopoietic recovery should be exhausted (causal therapy). This can range from G-CSF stimulation, to a trial of pulse-dose corticosteroids or anti-cytokine therapy (e.g. Anakinra, Tocilizumab), to a stem cell boost as a last resort in lymphoma patients who have undergone prior autologous stem cell transplantation. On the other hand, clinicians should be at high alert for infectious complications and adapt their infection surveillance and anti-infective prophylactic strategies accordingly. For example, therapeutic drug monitoring of posaconazole and meropenem levels may have revealed subtherapeutic dosing of these antiinfective agents in this patient [21]. The role of antifungal prophylaxis in the CAR $\mathrm{T}$-cell patient remains inconclusive. Adequately powered studies addressing the duration of neutropenia after CAR T-cell therapy, and potential clinical determinants of invasive fungal infections, will be critical to gauge the benefit of antifungal prophylaxis in this patient collective.

Candida species are a leading cause of fungusassociated morbidity and mortality in severely immunocompromised patients [21]. On day 41, this patient developed breakthrough C. glabrata Candidemia on micafungin, in the setting of both underlying colonization and neutropenic colitis. A recent report implicated the gastrointestinal tract as major source of echinocandin drug resistance in a murine model of $C$. glabrata colonization and systemic dissemination [22]. Of note, breakthrough Candida infections on micafungin may occur at the employed dose level $(50 \mathrm{mg} /$ day IV), suggesting that this dose may have been inadequate to prevent subsequent Candidemia [23]. Though cases of C. glabrata colitis are extremely rare [24], an opportunistic infection could not be ruled out given that C. glabrata infiltrates were discovered in enteral and colonic mucosal tissue on post-mortem examination. Importantly, the patients' clinical status deteriorated only after the stem cell boost resulted in signs of hematopoietic recovery. The paradoxical worsening of the pulmonary $A$. fumigatus infection is consistent with immune reconstitution inflammatory syndrome (IRIS), which has been previously described for HIV/AIDS [25] and for severely neutropenic patients with IPA [26]. In conclusion, this case emphasizes that infectious complications secondary to prolonged cytopenia influence non-relapse mortality after CAR T-cell therapy.

\section{Abbreviations}

ALC: Absolute Lymphocyte Count; ANC: Absolute Neutrophil Count; BM: Bone Marrow; CAR: Chimeric antigen receptor; CBC: Complete Blood Count; CRS: Cytokine release syndrome; CTCAE: Common Terminology Criteria for Adverse Events; DLBCL: Diffuse large B-cell lymphoma; FFP: Fresh frozen plasma; FLAIR: Fluid-attenuated inversion recovery; Flu/

Cy: Fludarabine/Cyclophosphamide; G-CSF: Granulocyte colony stimulating factor; Gl: Gastrointestinal; GM: Galactomannan; HSPCs: Hematopoietic stem and progenitor cells; ICANS: Immune effector cell-associated neurotoxicity syndrome; ICE-score: Immune Effector Cell-associated Encephalopathy score; ICU: Intensive care unit; IPA: Invasive pulmonary aspergillosis; LDH: Lactate Dehydrogenase; LYRIC: Lymphoma response to immunomodulatory therapy criteria; MDRD: Modification of Diet in Renal Disease; MPO: Myeloperoxidase; PAS: Periodic acid-Schiff; PBSC: Peripheral Blood Stem Cell; VRE: Vancomycinresistant Enterococcus; WBC: White Blood Count

\section{Acknowledgements}

Not applicable.

\section{Authors' contributions}

The original manuscript was written by KR. KR and WGK prepared the figures. MR performed the histological examination of the bone marrow. MS reviewed and edited the manuscript. The other authors (VB1, VB2, CS, PK, FS, $\mathrm{KD}, \mathrm{LVB}, J \mathrm{~S}, \mathrm{OW}, \mathrm{MD}, \mathrm{MvBB}$ ) contributed to patient management and participated in drafting and editing the manuscript. All authors read and approved the final manuscript.

\section{Funding}

Not applicable. Open Access funding enabled and organized by Projekt DEAL.

\section{Availability of data and materials}

The authors did not use any database, software, or tools for the writing of this manuscript.

\section{Ethics approval and consent to participate}

The authors obtained consent from the deceased patient's next-of-kin to participate.

\section{Consent for publication}

Written informed consent was obtained from the patient's next-of-kin for publication of this Case report and any accompanying images. A copy of the written consent is available for review by the Editor of this journal.

\section{Competing interests}

The authors declare that they have no competing interests related with this case report. 


\section{Author details}

'Department of Hematology and Oncology, University Hospital, LMU Munich, Munich, Germany. ${ }^{2}$ Laboratory for Translational Cancer Immunology, LMU Gene Center, Munich, Germany. ${ }^{3}$ German Cancer Consortium (DKTK) and German Cancer Research Center, Heidelberg, Germany. ${ }^{4}$ Department of Radiology, University Hospital, LMU Munich, Munich, Germany. ${ }^{5}$ Department of Pathology, University Hospital, LMU Munich, Munich, Germany. ${ }^{6}$ Department of Neurosurgery, University Hospital, LMU Munich, Munich, Germany. 'Department of Neurology, University Hospital, LMU Munich, Munich, Germany.

Received: 5 September 2020 Accepted: 28 December 2020 Published online: 28 January 2021

\section{References}

1. Nahas GR, Komanduri KV, Pereira D, Goodman M, Jimenez AM, Beitinjaneh $\mathrm{A}$, et al. Incidence and risk factors associated with a syndrome of persistent cytopenias after CAR-T cell therapy (PCTT). Leuk Lymphoma. 2020;61 (4):940-3.

2. Cordeiro A, Bezerra ED, Hirayama AV, Hill JA, Wu QV, Voutsinas J, et al. Late Events after Treatment with CD19-Targeted Chimeric Antigen Receptor Modified T Cells. Biol Blood Marrow Transplant. 2020;26(1):26-33.

3. Fried S, Avigdor A, Bielorai B, Meir A, Besser MJ, Schachter J, et al. Early and late hematologic toxicity following CD19 CAR-T cells. Bone Marrow Transplant. 2019;54(10):1643-50.

4. Schuster SJ, Bishop MR, Tam CS, Waller EK, Borchmann P, JP MG, et al. Tisagenlecleucel in Adult Relapsed or Refractory Diffuse Large B-Cell Lymphoma. N Engl J Med. 2019;380(1):45-56.

5. Locke FL, Ghobadi A, Jacobson CA, Miklos DB, Lekakis $\amalg$, Oluwole OO, et al. Long-term safety and activity of axicabtagene ciloleucel in refractory large B-cell lymphoma (ZUMA-1): a single-arm, multicentre, phase 1-2 trial. Lancet Oncol. 2019;20(1):31-42.

6. Donnelly JP, Chen SC, Kauffman CA, Steinbach WJ, Baddley JW, Verweij PE, et al. Revision and Update of the Consensus Definitions of Invasive Fungal Disease From the European Organization for Research and Treatment of Cancer and the Mycoses Study Group Education and Research Consortium. Clin Infect Dis. 2020;71(6):1367-76.

7. Lee DW, Santomasso BD, Locke FL, Ghobadi A, Turtle CJ, Brudno JN, et al. ASTCT Consensus Grading for Cytokine Release Syndrome and Neurologic Toxicity Associated with Immune Effector Cells. Biol Blood Marrow Transplant. 2019;25(4):625-38.

8. Valand HA, Huda F, Tu RK. Chimeric Antigen Receptor T-Cell Therapy: What the Neuroradiologist Needs to Know. AJNR Am J Neuroradiol. 2019;40(5):766-8

9. Saberi A, Roudbary SA, Ghayeghran A, Kazemi S, Hosseininezhad M. Diagnosis of Meningitis Caused by Pathogenic Microorganisms Using Magnetic Resonance Imaging: A Systematic Review. Basic Clin Neurosci. 2018:9(2):73-86.

10. Taplitz RA, Kennedy EB, Bow EJ, Crews J, Gleason C, Hawley DK, et al. Antimicrobial Prophylaxis for Adult Patients With Cancer-Related Immunosuppression: ASCO and IDSA Clinical Practice Guideline Update. J Clin Oncol. 2018:36(30):3043-54.

11. Sterner RM, Sakemura R, Cox MJ, Yang N, Khadka RH, Forsman CL, et al. GMCSF inhibition reduces cytokine release syndrome and neuroinflammation but enhances CAR-T cell function in xenografts. Blood. 2019;133(7):697-709.

12. Rodrigues CF, Silva S, Henriques M. Candida glabrata: a review of its features and resistance. Eur J Clin Microbiol Infect Dis. 2014;33(5):673-88.

13. Whaley SG, Berkow EL, Rybak JM, Nishimoto AT, Barker KS, Rogers PD. Azole Antifungal Resistance in Candida albicans and Emerging Non-albicans Candida Species. Front Microbiol. 2016;7:2173.

14. Wudhikarn K, Pennisi M, Garcia-Recio M, Flynn JR, Afuye A, Silverberg ML, et al. DLBCL patients treated with CD19 CAR T cells experience a high burden of organ toxicities but low nonrelapse mortality. Blood Adv. 2020; 4(13):3024-33

15. Hill JA, Li D, Hay KA, Green ML, Cherian S, Chen X, et al. Infectious complications of CD19-targeted chimeric antigen receptor-modified T-cell immunotherapy. Blood. 2018;131(1):121-30.

16. Park JH, Romero FA, Taur Y, Sadelain M, Brentjens RJ, Hohl TM, et al. Cytokine Release Syndrome Grade as a Predictive Marker for Infections in Patients With Relapsed or Refractory B-Cell Acute Lymphoblastic Leukemia
Treated With Chimeric Antigen Receptor T Cells. Clin Infect Dis. 2018;67(4): $533-40$.

17. Haidar G, Dorritie K, Farah R, Bogdanovich T, Nguyen MH, Samanta P. Invasive Mold Infections After Chimeric Antigen Receptor-Modified T-cell Therapy: A Case Series, Review of the Literature, and Implications for Prophylaxis. Clin Infect Dis. 2020;71(3):672-6.

18. Wudhikarn K, Palomba ML, Pennisi M, Garcia-Recio M, Flynn JR, Devlin SM et al. Infection during the first year in patients treated with CD19 CAR T cells for diffuse large B cell lymphoma. Blood Cancer J. 2020;10(8):79.

19. Luo H, Wang N, Huang L, Zhou X, Jin J, Li C, et al. Inflammatory signatures for quick diagnosis of life-threatening infection during the CAR T-cell therapy. J Immunother Cancer. 2019;7(1):271.

20. Cain DW, Cidlowski JA. Immune regulation by glucocorticoids. Nat Rev Immunol. 2017;17(4):233-47.

21. Chen L, Wang Y, Zhang T, Li Y, Meng T, Liu L, et al. Utility of posaconazole therapeutic drug monitoring and assessment of plasma concentration threshold for effective prophylaxis of invasive fungal infections: a metaanalysis with trial sequential analysis. BMC Infect Dis. 2018;18(1):155.

22. Healey KR, Nagasaki Y, Zimmerman M, Kordalewska M, Park S, Zhao Y, et al. The Gastrointestinal Tract Is a Major Source of Echinocandin Drug Resistance in a Murine Model of Candida glabrata Colonization and Systemic Dissemination. Antimicrob Agents Chemother. 2017;61(12): e01412-7.

23. van Burik JA, Ratanatharathorn V, Stepan DE, Miller CB, Lipton JH, Vesole $\mathrm{DH}$, et al. Micafungin versus fluconazole for prophylaxis against invasive fungal infections during neutropenia in patients undergoing hematopoietic stem cell transplantation. Clin Infect Dis. 2004;39(10):1407-16.

24. Jimenez Rosales R, CAB AC, Ojeda Hinojosa M. The first reported case of colonic infection caused by Candida glabrata. Rev Esp Enferm Dig. 2019; 111(8):648.

25. Muller M, Wandel S, Colebunders R, Attia S, Furrer H, Egger M, et al. Immune reconstitution inflammatory syndrome in patients starting antiretroviral therapy for HIV infection: a systematic review and metaanalysis. Lancet Infect Dis. 2010;10(4):251-61.

26. Jung J, Hong HL, Lee SO, Choi SH, Kim YS, Woo JH, et al. Immune reconstitution inflammatory syndrome in neutropenic patients with invasive pulmonary aspergillosis. J Infect. 2015;70(6):659-67.

\section{Publisher's Note}

Springer Nature remains neutral with regard to jurisdictional claims in published maps and institutional affiliations.

Ready to submit your research? Choose BMC and benefit from:

- fast, convenient online submission

- thorough peer review by experienced researchers in your field

- rapid publication on acceptance

- support for research data, including large and complex data types

- gold Open Access which fosters wider collaboration and increased citations

- maximum visibility for your research: over $100 \mathrm{M}$ website views per year

At $\mathrm{BMC}$, research is always in progress.

Learn more biomedcentral.com/submissions 